\title{
Avaliação de desempenho de Heavy Hitters utilizando P4 e XDP
}

\author{
Douglas L. Machado, Ariel G. de Castro, Francisco G. Vogt, Marcelo C. Luizelli \\ ${ }^{1}$ Universidade Federal do Pampa (UNIPAMPA)
}

Aplicações de monitoramento de infraestruturas de redes são cruciais para a correta operação e identificação de comportamentos e anomalias. Muitas aplicações de monitoramento se beneficiam da identificação dos fluxos de rede que mais consomem recursos (por exemplo, largura de banda) nas infraestruturas para identificar ataques, congestionamentos e para a engenharia de tráfego - apenas para mencionar alguns exemplos [Sivaraman et al. 2017]. Os fluxos de rede com o maior volume de dados são conhecidos como Heavy Hitters. A identificação dos Heavy Hitters consiste na contagem dos fluxos que mais contribuem para a utilização dos recursos nas infraestruturas [Ben Basat et al. 2017].

Com a consolidação das redes definidas por software (SDN - Software-defined Networks), a identificação de Heavy Hitters tem o potencial de ser feita de maneira online pelo próprio plano de dados [Sivaraman et al. 2017]. Ao se utilizar a identificação de $\mathrm{He}$ avy Hitters no plano de dados, elimina-se a sobrecarga da transferência contínua de dados entre os planos de dados e de controle. Para além disso, a contagem dos fluxos tende a ser mais precisa que abordagens tradicionais de monitoramento baseadas em protocolos como, por exemplo, NetFlow ou SFlow. A programabilidade do plano de dados permite redefinir o processamento dos pacotes nos dispositivos, incluindo o suporte a novos protocolos e funcionalidades. A programabilidade do plano de dados emergiu recentemente com a consolidação da linguagem P4 [Bosshart et al. 2014] e de dispositivos de encaminhamento programáveis (por exemplo, Barefoot Tofino e SmartNICS). A linguagem P4 é independente de protocolo (isto é, os protocolos são definidos pelo programador) e independente de arquitetura (isto é, o compilador utilizado gera um código objeto de acordo com a arquitetura utilizada).

Outra abordagem recente para programabilidade do plano de dados é o XDP $-e X$ press Data Path [Høiland-Jørgensen et al. 2018]. Ao contrário das aplicações escritas em P4 e compiladas para um hardware independente, XDP é um plano de dados baseado em eBPF (Extended Berkeley Packet Filter) [Xhonneux et al. 2018] executado diretamente no Kernel do Linux, disponível a partir da versão 4.8. A ideia do XDP consiste em adicionar um mecanismo de decisão baseado em eBPF logo após o pacote ser recebido pelo driver da interface de rede. Dessa forma, o pacote recebido pode ser tratado de maneira prioritária e personalizada pelo módulo de rede do sistema operacional, sem a necessidade de alocação prévia de memória para o pacote (o que é uma operação custosa), nem cópias desnecessárias entre o Kernel e o espaço de usuário.

Neste trabalho, objetiva-se conduzir uma avaliação de desempenho de algoritmos para identificar Heavy Hitters utilizando P4 e XDP e os algoritmos propostos por [Metwally et al. 2005, Ben Basat et al. 2017]. Pretende-se escrever uma aplicação de encaminhamento de pacotes que realize a contagem dos pacotes de acordo com os algoritmos de Heavy Hitter, utilizando como chave de identificação da contagem os endereços 
IPs (origem/destino). Como a quantidade de pares de endereços IPs é exponencial, esperase empregar estruturas de dados eficientes espacialmente como, por exemplo, Counting Bloom Filter. O programa escrito em P4 será compilado para ser executado em uma interface de rede programável (por exemplo, SmartNIC Netronome) e diretamente no Kernel do Linux a partir do compilador P4C-XDP. Com a avaliação, pretende-se estimar os custos em termos de pacotes processados por segundo, latência e vazão atingidas - as quais podem ser impactadas de acordo com a implementação do algoritmo de Heavy Hitter utilizado. Para além disso, pretende-se identificar os limites de utilização de memória em ambas as implementações e o consumo de ciclos de CPU.

\section{Referências}

Ben Basat, R., Einziger, G., Friedman, R., Luizelli, M. C., and Waisbard, E. (2017). Constant time updates in hierarchical heavy hitters. In Proceedings of the Conference of the ACM Special Interest Group on Data Communication, SIGCOMM '17, pages 127-140, New York, NY, USA. ACM.

Bosshart, P., Daly, D., Gibb, G., Izzard, M., McKeown, N., Rexford, J., Schlesinger, C., Talayco, D., Vahdat, A., Varghese, G., and Walker, D. (2014). P4: Programming protocol-independent packet processors. SIGCOMM Comput. Commun. Rev., 44(3):87-95.

Høiland-Jørgensen, T., Brouer, J. D., Borkmann, D., Fastabend, J., Herbert, T., Ahern, D., and Miller, D. (2018). The express data path: Fast programmable packet processing in the operating system kernel. In Proceedings of the 14th International Conference on Emerging Networking EXperiments and Technologies, CoNEXT '18, pages 54-66, New York, NY, USA. ACM.

Metwally, A., Agrawal, D., and El Abbadi, A. (2005). Efficient computation of frequent and top-k elements in data streams. In Proceedings of the 10th International Conference on Database Theory, ICDT'05, pages 398-412, Berlin, Heidelberg. SpringerVerlag.

Sivaraman, V., Narayana, S., Rottenstreich, O., Muthukrishnan, S., and Rexford, J. (2017). Heavy-hitter detection entirely in the data plane. In Proceedings of the Symposium on SDN Research, SOSR '17, pages 164-176, New York, NY, USA. ACM.

Xhonneux, M., Duchene, F., and Bonaventure, O. (2018). Leveraging ebpf for programmable network functions with ipv6 segment routing. In Proceedings of the 14th International Conference on Emerging Networking EXperiments and Technologies, CoNEXT'18, pages 67-72, New York, NY, USA. ACM. 